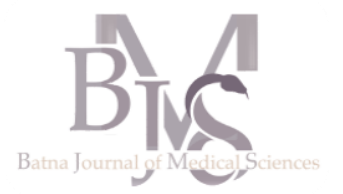

Syndrome des antiphospholipides : comment prendre en charge la grossesse ?

\title{
Antiphospholipid syndrome: How to manage pregnancy?
}

\author{
Mounir Moukit, Jaouad Kouach, Rachid Aitbouhou, Moulay El Mehdi El Hassani, \\ Abdellah Babahabib, Driss Moussaoui Rahali, Mohammed Dehayni
}

Service de Gynécologie et Obstétrique, Hôpital Militaire d'Instruction Mohammed V, Rabat, Maroc.

\section{Correspondance à :}

Mounir MOUKIT

moukitmounir@yahoo.com

DOI :https://doi.org/10.48087/ BJMSra.2017.4203

Il s'agit d'un article en libre accès distribué selon les termes de la licence Creative Commons Attribution International License (CC BY 4.0), qui autorise une utilisation, une distribution et une reproduction sans restriction sur tout support ou format, à condition que l'auteur original et la revue soient dûment crédités.

\section{Pour citer l'article :}

Moukit M, Kouach J, Aitbouhou R, et al. Syndrome des antiphospholipides : comment prendre en charge la grossesse ? Batna J Med Sci 2017;4(2):139-144. https://doi.org/10.48087/ BJMSra.2017.4203

\section{RÉSUMÉ}

La survenue d'une grossesse au cours du syndrome des antiphospholipides est une situation qui reste associée à une morbi-mortalité maternelle et fœtale plus importante que dans la population générale. L'amélioration de la prise en charge de ces grossesses est conditionnée par leur planification systématique et par une prise en charge multidisciplinaire incluant obstétricien, interniste et anesthésiste. Un traitement compatible avec la grossesse, adapté aux antécédents et aux risques, ainsi qu'une surveillance régulière plus rapprochée en fin de grossesse, sont les principaux éléments permettant une issue favorable de ces grossesses à haut risque. Cet article a pour objectif de proposer une mise au point sur la prise en charge actuelle de la grossesse au cours du syndrome des antiphospholipides afin de limiter le risque de complications et d'assurer le meilleur pronostic tant maternel que fotal.

Mots-clés : grossesse; syndrome des antiphospholipides; complications obstétricales; traitement.

\section{INTRODUCTION}

Comme pour les maladies auto-immunes, le syndrome des antiphospholipides (SAPL) touche plus fréquemment la femme jeune, et la problématique de la grossesse va donc se poser. Cette fois $\mathrm{ci}$, la situation est plus complexe vu l'absence de recommandations internationales. Actuellement, on ne dispose que d'avis d'experts ou de consensus nationaux. Même si le pronostic de ces grossesses s'est nettement amélioré, celles-ci restent des grossesses à risque et l'optimisation de leur prise en charge est un véritable challenge nécessitant une collaboration étroite entre les différents intervenants (obstétricien, interniste, anesthésiste). Dans cette mise au point, et après un rappel sur cette pathologie thrombophilique acquise, nous aborderons successivement les différentes complica-tions liées au syndrome des anticorps antiphospholipides au cours de la grossesse et nous proposerons une prise en charge pratique, basée sur les résultats des grandes séries publiées ainsi que sur notre propre expérience.

\section{GÉNÉRALITÉS}

Le SAPL appartient au groupe des maladies auto-immunes et représente un état thrombo-

\begin{abstract}
Pregnancy in patients with antiphospholipid syndrome is a situation that remains associated with higher maternal and fetal mortality/morbidity than in the general population. Improving the care of these pregnancies depends upon a systematic pregnancy planning and a multidisciplinary approach including obstetrician, internist and anesthetist. The use of appropriate medications during pregnancy adjusted to the patient's medical history and risk factors, and a regular monitoring are the best tools for a favorable outcome for these high-risk pregnancies. The aim of this article is to perform an update on the medical care of pregnancy during antiphospholipid syndrome to reduce the risk of complications and to ensure the best maternal and fetal prognosis.
\end{abstract}

Keywords: pregnancy; antiphospholipid syndrome; adverse obstetrical outcome; management.

philique acquis dû à la présence d'autoanticorps antiphospholipides (APL) [1]. Il peut être rencontré en dehors de tout cadre pathologique défini (SAPL primaire) ou associé à une des grandes connectivites (SAPL secondaire), essentiellement le lupus érythémateux systémique (LES). Il se manifeste par des thromboses, qui surviennent sur des parois vasculaires saines et peuvent toucher tous les territoires vasculaires quels que soient leurs types ou leurs calibres ainsi que par des manifestations obstétricales, comprenant des fausses couches spontanées (FCS), des morts fotales in utero (MFIU), des restrictions de croissance intra-utérine (RCIU) et des prématurités, mais aussi des pré-éclampsies (PE) et des éclampsies [2]. En effet, les anticorps APL sont la cause directe de ces manifestations thrombotiques et obstétricales. Ils désignent un groupe hétérogène d'autoanticorps qui recon-naissent comme antigène soit directement les phospholipides membranaires (PL), soit des protéines se fixant sur les PL comme la $\beta 2$-glycoprotéine I ( $\beta 2 \mathrm{GPI})$ ou la pro-thrombine. Les principaux APL sont le lupus anticoagulant (LA), les anti- $\beta 2$ GPI et les anti-cardiolipines. Plusieurs autres APL existent, comme l'anti-prothrombine, l'antiphosphatidylsérine et l'anti-annexine V. Tous n'ont pas la même pathogénicité et les anticorps anti- $\beta 2 \mathrm{GPI}$ dirigés contre le domaine 
I de la glycoprotéine semblent être ceux essentiellement responsables des manifestations thrombotiques et obstétricales. Cependant, les APL ne sont pas spécifiques du SAPL et peuvent se rencontrer en association à diverses infections (syphilis, lèpre, hépatites virales, VIH, EBV, CMV), à certaines hémopathies notamment lymphoïdes et à certains traitements (chlorpromazine, $\beta$-bloquants, quinidiniques et interférons), voire chez des individus sains. Ces APL ne sont généralement pas thrombogènes. Ils sont souvent transitoires et détectés à faible taux [1].

\section{CRITÈRES DIAGNOSTIQUES}

Diagnostiquer le SAPL est donc complexe, et a fait l'objet d'une première conférence de consensus en 1998 à Sapporo, au Japon, au cours de laquelle des critères de classification préliminaires ont été définis [3]. Le diagnostic était confirmé en présence d'une thrombose (veineuse, artérielle ou microcirculatoire) ou d'une complication obstétricale, associée à la présence d'un APL, soit anti-cardiolipine (IgG ou IgM) soit LA, persistant au-delà de 6 semaines et à taux modéré à élevé. Ces critères ont été révisés en 2006 lors du 11 ème congrès international des APL à Sydney en Australie (Tableau 1) [4]. Les principales modifications sont la prise en compte des anti- $\beta 2$ GPI (IgG ou IgM) et la nécessité d'une persistance de la positivité des APL au-delà de 12 semaines. La prise en compte des APL seulement en cas de persistance au-delà de 12 semaines et non plus 6 semaines ne semble pas faire de différence, en tout cas ne récuse pas le diagnostic de SAPL quand les nouveaux critères de classification sont appliqués aux patients antérieurement diagnostiqués sur la base des critères de Sapporo [5]. Au contraire, ces nouveaux critères augmentent la performance diagnostique, en particulier en milieu obstétrical, du fait de la prise en compte des anti- $\beta 2$ GPI $[5,6]$.

\section{INDICATION D'UNE BIOLOGIE ANTIPHOSPHOLIPIDES}

Il est indispensable de rechercher l'existence d'une biologie APL chez une femme lupique avec un désir de grossesse, de même chez une femme ayant une anomalie faisant évoquer un SAPL (antécédent de FCS répétées ou d'MFIU inexpliquée, de thrombose vasculaire, notion de TCA spontanément allongé, livedo pathologique, valvulopathie cardiaque inexpliquée, thrombopénie périphérique, VDRL positif dissocié). En effet, dans ce contexte, la biologie APL est généralement présente et justifie une prise en charge adaptée [7]. Des manifestations cliniques évocatrices d'un SAPL obstétrical peuvent être observées en l'absence de biologie antiphospholipides et le terme de SAPL séronégatif a été proposé [8].

\section{RISQUES AU COURS DE LA GROSSESSE}

Selon le mode d'entrée dans la maladie que ce soit par des FCS, par une mort fœtale ou par un événement thrombotique, l'évolution n'est pas comparable et les risques obstétricaux non plus. Dans L'étude de Bramham et al. le pronostic obstétrical était significativement plus mauvais dans le groupe 3 (SAPL avec antécédent de thrombose) par rapport au groupe 1 (SAPL avec antécédents de FCS répétées) et au groupe 2 (SAPL avec antécédents de MFIU ou de prématurité) malgré un traitement adapté [9].
Tableau 1. Critères diagnostiques révisés du SAPL (Sydney 2006).

Critères cliniques:
Thrombose vasculaire:
Au moins un épisode thrombotique artériel, veineux ou des petits
vaisseaux, touchant tout tissu ou organe. La thrombose doit être
confirmée par un critère objectif et validé (aspect typique à
l'imagerie ou par un examen anatomopathologique, sans
inflammation vasculaire sous-jacente).

\section{Manifestations obstétricales:}

Au moins une MFIU inexpliquée, après la 10 $\mathrm{SA}$ avec morphologie fœtale normale documentée par une échographie ou par examen macroscopique.

Au moins une naissance prématurée avant la $34^{\mathrm{e}} \mathrm{SA}$, d'un foetus morphologiquement normal, en rapport avec une PE sévère, une éclampsie, ou une insuffisance placentaire sévère.

Au moins 3 fausses couches consécutives et inexpliquées avant la $10^{\mathrm{e}} \mathrm{SA}$, après exclusion d'une anomalie anatomique ou hormonale maternelle et d'une anomalie chromosomique maternelle ou paternelle.

\begin{abstract}
Critères biologiques:
Lupus anticoagulant présent à au moins 2 reprises, à 12 semaines d'intervalle, selon les recommandations de l'ISTH.

Anticorps anticardiolipines (IgG et/ou IgM) présents à au moins 2 reprises, à un titre intermédiaire ou élevé ( $>$ 40UGPL ou UMPL, ou $>99$ e percentile), mesuré par une technique Elisa standardisée.

Anticorps anti $\beta 2$-glycoprotéine I (IgG ou IgM) présents à un titre $>$ au 99 e percentile, à au moins 2 reprises, à 12 semaines d'intervalle selon une technique Elisa standardisée.

SAPL: syndrome des antiphospholipides; MFIU: mort fœtale in utéro; SA: semaine d'aménorrhée; PE: pré-éclampsie; ISTH: International Society on Thrombosis and Haemostasis; Ig: immunoglobuline. Présence d'un syndrome des antiphospholipides si au moins un critère clinique et un critère biologique sont présents.
\end{abstract}

\section{Risques foetaux}

Dominés par les FCS, la MFIU, l'insuffisance placentaire et la prématurité. Ces risques sont diminués par une prise en charge thérapeutique adaptée.

Fausses couches spontanées répétées et morts fotales in utero: les FCS précoces avant la 10ème semaine d'aménorrhée (SA) sont fréquentes dans la population générale mais leur caractère récurrent est plus rare. Dans cette situation, 10 à $20 \%$ des femmes ont une biologie APL [10]. Les FCS tardives (entre la 10 ème et la 22 ème SA) et la mort fœtale in utero inexpliquée (définie par le décès d'un fœtus morphologiquement normal entre 22 SA et le début du travail, ou par un poids fœtal supérieur à $500 \mathrm{~g}$ si l'âge gestationnel est inconnu) sont beaucoup plus rares dans la population générale.

L'insuffisance placentaire : définie par la présence d'une restriction de croissance intra-utérine, d'un oligoamnios, d'anomalies du doppler ombilical ou du rythme cardiaque fœtal. Une analyse de population de 141.286 grossesses en Floride retrouve une corrélation entre la positivité des APL et l'insuffisance placentaire, odd ratio ajusté aux autres facteurs de risque de 4,58 [11]. Ce risque augmente fortement en cas de LES associé (odd ratio ajusté de 14,12 en cas d'association LES et APL, contre 4,06 pour la positivité isolée des APL) [11]. 
La prématurité : définie comme une naissance vivante survenant entre $22 \mathrm{SA}$ et $36 \mathrm{SA}+6 \mathrm{j}$. Les insuffisances placentaires avec RCIU et/ou oligoamnios justifiant un déclenchement de l'accouchement sont fréquemment rapportées. Cela explique en grande partie l'importance de la prématurité qui est de ce fait induite au cours du SAPL avec une fréquence variant de 32 à $65 \%$ [10,12] .

\section{Risques maternels}

Les complications maternelles attribuables au SAPL sont l'hypertension artérielle gravidique (HTAG), la PE, l'éclampsie, l'hématome rétroplacentaire (HRP), le syndrome de HELLP et la survenue d'un événement thromboembolique, parfois dans le contexte d'un syndrome catastrophique des antiphospholipides (SCAP).

Hypertension artérielle gravidique : la prévalence de l'HTA gravidique sans protéinurie au cours du SAPL est mal évaluée, mais affecte de manière négative le devenir de la grossesse. Dans son étude cas-témoins, Yamada retrouvait en analyse multivariée une association statistiquement significative entre l'IgG anti-cardiolipine et l'HTAG (odds ratio 11,4), ainsi qu'entre la double positivité pour le LA et l'anticardiolipine et l'HTAG sévère (odds ratio 250) [13].

Pré-éclampsie et éclampsie : la PE (définie par une PAS $\geq$ $140 \mathrm{mmHg}$ et/ou une $\mathrm{PAD} \geq 90 \mathrm{mmHg}$, associée à une protéinurie $\geq 0,3 \mathrm{~g} / 24 \mathrm{~h}$ ) et l'éclampsie (crises convulsives suivies d'un état comateux) sont des complications classiques $\mathrm{du}$ SAPL. Dans la série Euro-Phospholipid, sur les 590 patientes ayant présenté au moins une grossesse avant l'inclusion, 9,5\% avaient déclenché une PE et 4,4\% une éclampsie [14]. Dans l'analyse de population de Nodler, la présence d'APL était associée à la PE ou l'éclampsie avec un odds ratio ajusté aux autres facteurs de risque de 2,93 [11]. Dans cette dernière, la présence d'un LES en plus de la positivité des APL renforçait cette association (odds ratio ajusté de 33,14, contre 2,12 pour la positivité isolée des APL) [11]. Concernant le risque de chaque APL, la méta-analyse d'Abou-Nassar retrouve pour le LA un odds ratio de 2,34 dans les études cas-témoins, pour les anti-cardiolipines (IgG ou IgM, >5 GPL/MPL) un odds ratio de 1,52 dans les études castémoins avec pour un IgG anti-cardiolipine $>20$ GPL un odds ratio de 2,20 dans les études de cohortes, et pour les anti$\beta 2 \mathrm{GPI}$ (IgG ou IgM, >5 GPL/MPL) un odds ratio de 19,14 dans les études de cohorte avec pour l'isotype IgG un odds ratio de 24 dans ces mêmes études [15]. Le caractère précoce (dès 15 à $16 \mathrm{SA}$ ) ou sévère de la PE est évocateur d'un SAPL [16]. En cas de SAPL secondaire à un LES, il est parfois difficile de différencier une PE d'une poussée lupique. La présence de manifestations lupiques extra-rénales, d'une hématurie et une baisse des fractions C3 et C4 du complément (d'autant plus que les fractions du complément augmentent physiologiquement au cours de la grossesse) sont autant d'éléments en faveur d'une glomérulonéphrite lupique.

Hématome rétroplacentaire : l'HRP était rapporté chez $2 \%$ des 590 patientes ayant présenté au moins une grossesse à l'inclusion dans la cohorte Euro-Phospholipid [15]. Il existe peu de données sur cette complication au cours du SAPL. La méta-analyse d'Abou-Nassar ne permet pas de relier l'HRP à un APL particulier [16].
Syndrome de HELLP: le syndrome de HELLP (H: Hemolysis, EL: Elevated Liver enzymes, LP: Low Platelet count) est considéré comme une variante ou une forme compliquée de la PE. Occasionnellement, ce syndrome peut compliquer l'évolution d'un LES ou d'un SAPL $[17,18]$. Son incidence au cours du SAPL est difficile à estimer. Une méta-analyse publiée entre 1994 et 2010 ne retrouvait que 51 grossesses de 50 patientes présentant des APL (SAPL défini pour 40 d'entre elles) compliquées de syndrome de HELLP [19]. La plupart des cas survenait pendant le 2 ème ou le 3ème trimestre de la grossesse mais certaines patientes déclaraient ce syndrome en post-partum. Il est souvent plus précoce et plus sévère et peut être un des modes de révélation du SAPL [18].

Thrombose maternelle : la fréquence des thromboses maternelles au cours de la grossesse chez les patientes atteintes de SAPL est mal évaluée, mais semble rare dans notre expérience surtout lorsque le traitement est bien adapté, sauf au cours du SCAP défini par l'atteinte d'au moins 3 organes dans un laps de temps très court (moins d'une semaine) avec confirmation histologique de la présence de thrombi dans les capillaires $[7,20]$. Le registre international du SCAP recense 15 cas survenus pendant la grossesse ou en post-partum ou post-abortum [21]. Les mères avaient une prévalence élevée d'antécédents de pertes fotales. Un syndrome de HELLP était associé dans 53 $\%$ des cas et un infarctus placentaire dans $27 \%$ des cas; la mortalité maternelle était de $46 \%$ et la mortalité fœetale de $54 \%$ [21]. D'autres facteurs peuvent précipiter sa survenue tels qu'une infection, une poussée lupique ou une interruption de l'anticoagulation au cours du travail [22].

\section{Risques liés au traitement}

La toxicité des médicaments fait également partie des complications surtout quand il s'agit d'un SAPL secondaire associé à un lupus. En pratique, les complications hémorragiques sous traitement anticoagulant ou antiagrégant sont rarement observées, de même que l'ostéoporose sous héparine et la thrombopénie immunoallergique à l'héparine [7].

\section{Infertilité}

Le lien entre SAPL et infertilité reste discuté [23]. Cependant, plusieurs travaux ont montré que la stérilité primaire était associée à une prévalence plus élevée d'APL. Kovacs et al. rapporte une série de 100 patientes stériles ou infertiles, pour lesquelles toutes les autres causes avaient été écartées [24]. Ces patientes bénéficiaient alors d'un bilan auto-immun comprenant la recherche des anti-cardiolipines, des anti- $32 \mathrm{GPI}$, des anti-annexine $\mathrm{V}$, des anti-prothrombines et des anti-phosphatidylsérine, ainsi que des FAN, des antiDNA, des anti-ENA, des anti-TPO et des anticorps antispermatozoïde. Le LA n'était pas recherché. Au total, 51 patientes possédaient au moins un APL détecté au moins une fois. Trente et une de ces 51 patientes ont pu développer une grossesse, après un traitement par aspirine seule ou en association à une HBPM ou une corticothérapie, bien que 5 de ces grossesses se soient soldées par une fausse couche [24]. 


\section{FACTEURS PRONOSTIQUES OBSTÉTRICAUX}

Facteurs cliniques : Dans l'étude de Ruffatti et al., menée chez 410 grossesses avec SAPL, une complication est survenue au cours de 57 grossesses [25]. Les facteurs de risque cliniques retrouvés étaient l'association à un lupus systémique ou à une autre maladie auto-immune et l'antécédent de thrombose ou de complication au cours d'une grossesse antérieure [25]. Les patientes ayant un SAPL se manifestant par des FCS récurrentes ont habituellement un bon pronostic lorsque le premier trimestre de la grossesse est passé, par rapport aux patientes ayant des antécédents thrombotiques (même avec traitement adapté) [9]. Ces résultats sont conformes à notre expérience où l'existence d'antécédents de thrombose ou d'un SAPL secondaire était des éléments de mauvais pronostic.

Facteurs biologiques : Les différents anticorps ne confèrent également pas tous le même risque obstétrical. Il est établi que l'association d'un lupus anticoagulant, d'anticorps anti- $\beta 2$ GPI et anticardiolipine (triple positivité APL) confère un risque élevé d'accidents obstétricaux [25]. Cependant, dans l'étude américaine PROMISSE, la présence d'un lupus anticoagulant était un élément de mauvais pronostic avec $41 \%$ de complications obstétricales versus aucune chez les patientes sans anticoagulant circulant, et il n'y avait aucun lien entre le pronostic obstétrical et l'existence de taux élevés d'anticardiolipine ou d'anti- $\beta 2$ GPI [26].

Facteurs échographiques : Au cours du Doppler effectué au deuxième trimestre, la présence d'un index de résistance utéro-placentaire anormalement élevé, la persistance d'une incisure protodiastolique (ou notch) et la diminution du flux diastolique sont considérées comme des éléments péjoratifs [27].

\section{PRISE EN CHARGE}

Il n'existe pas de consensus clair sur la prise en charge thérapeutique des grossesses au cours du SAPL, du fait de l'absence de grandes études prospectives randomisées. Les recommandations découlent donc plus d'opinion d'experts. L'objectif est de prévenir les complications à la fois foetales et maternelles. Avec un traitement approprié, plus de $70 \%$ des femmes enceintes atteintes de SAPL mènent une grossesse à terme [9].

La consultation pré-conceptionnelle : La grossesse doit être précédée d'une consultation pré-conceptionnelle qui permet d'une part, d'éliminer les contre-indications à la grossesse (Tableau 2) et d'autre part, d'informer le couple sur les risques gravidiques, d'adapter les traitements et d'organiser un suivi multidisciplinaire incluant obstétricien et interniste. Cette consultation pré-conceptionnelle permet de remettre l'ordonnance du traitement à la patiente. Celui-ci sera débuté dès la grossesse connue.

Protocole thérapeutique pour la prévention des complications obstétricales : La prévention des complications obstétricales du SAPL repose sur la mise en œuvre de protocole décidé en fonction du type de SAPL (thrombotique, ou obstétrical pur) et des antécédents obstétricaux. Les moyens thérapeutiques disponibles sont : les antiagrégants plaquettaires (aspirine à faible dose), les anticoagulants (héparine de bas poids moléculaire ou héparine non fractionnée), les corticoïdes et l'Hydroxychloroquine (Plaquenil ${ }^{\circledR}$ ). L'intensité et la durée du traitement anticoagu-
Tableau 2. Contre-indications à la grossesse au cours du syndrome des antiphospholipides.

Existence d'une hypertension artérielle pulmonaire sévère.

Hypertension artérielle sévère et non contrôlée.

Valvulopathie mal tolérée.

Antécédent thrombotique majeur et récent.

Insuffisance rénale sévère (créatinémie $>140$ mmol/l)

lant font encore l'objet de nombreuses discussions. Le protocole thérapeutique adopté dans notre formation, qui est basé sur les grandes séries publiées ainsi que sur notre propre expérience, est résumé dans la figure 1.

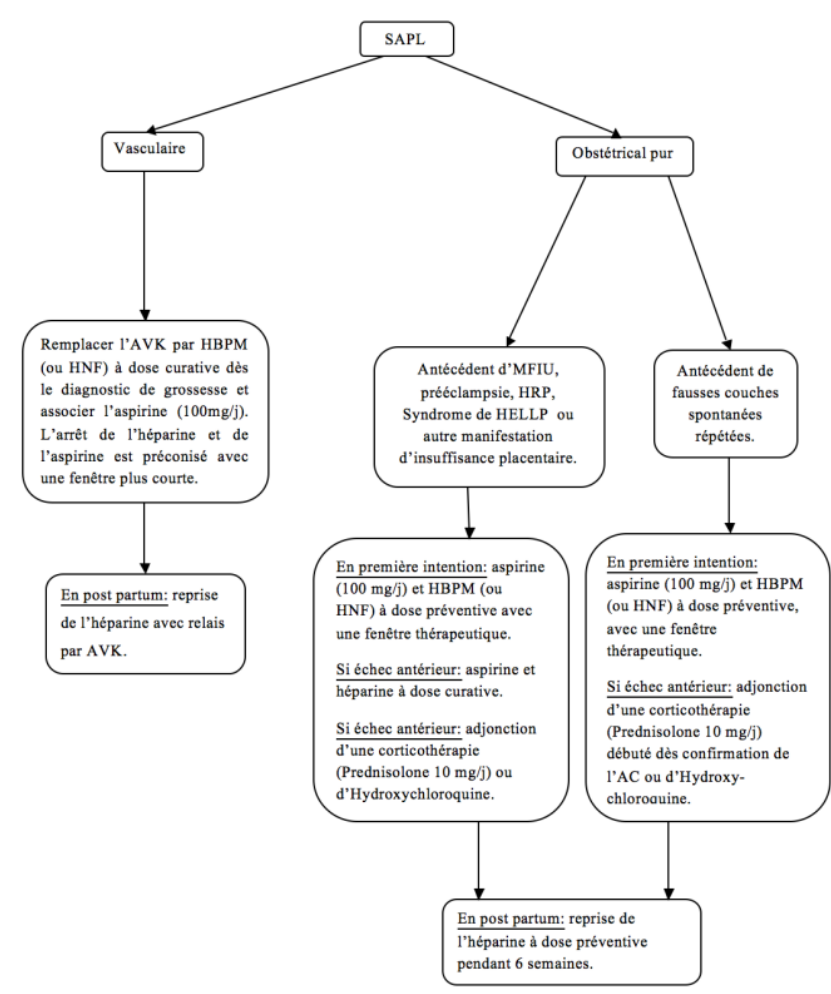

Figure 1. Recommandations thérapeutiques en fonction du type de SAPL et des antécédents obstétricaux.

Rappelons que l'utilisation moins contraignante et le risque moins élevé de thrombopénie et d'ostéoporose sous héparine de bas poids moléculaire (HBPM) les font souvent préférer à l'héparine non fractionnée (HNF). En ce qui concerne le SAPL obstétrical avec antécédent de fausses couches récidivantes, deux essais prospectifs ont souligné que l'association héparine et aspirine à faible dose améliorait considérablement le pronostic fœtal par rapport à l'aspirine seule $[28,29]$. De plus, une méta-analyse a montré que l'association héparine et aspirine était très largement supérieure à l'aspirine seule, pour la prévention des pertes fœetales chez les patients avec un SAPL [30]. Cependant, ce sujet reste suffisamment controversé pour que quelques experts considèrent que l'option d'une monothérapie par aspirine reste également possible [31]. L'attitude à tenir vis-à-vis du SAPL obstétrical avec antécédent d'MFIU, de PE, de syndrome de HELLP ou d'une autre manifestation d'insuffisance placentaire est beaucoup moins claire. Les seules données 
proviennent des études faites sur les pertes fœtales dans lesquelles ces patientes sont parfois incluses. La plupart des experts recommandent d'associer héparine et aspirine [31]. Nous proposons de débuter l'aspirine avant la conception, et d'introduire l'héparine dès la confirmation d'une grossesse intra-utérine même si l'on ne dispose d'aucune recommandation explicite à ce sujet dans la littérature. La dose optimale d'héparine (préventive ou curative) n'a été que très partiellement étudiée dans les différentes études sur les pertes fœtales. En pratique, chez une patiente ayant un SAPL défini, avec événements obstétricaux antérieurs survenus en l'absence de traitement, nous proposons l'association d'aspirine à faible dose avec une héparine à dose préventive (une injection quotidienne). Chez une patiente qui a eu une perte fœtale ou une autre complication obstétricale avec cette association, une héparinothérapie à dose curative (deux injections quotidiennes) peut être proposée. En raison de modifications du volume de distribution de l'héparine en cours de la grossesse, il paraît justifié de suivre l'efficacité de cette anticoagulation en dosant régulièrement l'activité anti-Xa et en adaptant si besoin l'héparinothérapie [32]. L'objectif est l'obtention d'une activité anti-Xa efficace (entre 0,5 et $1 \mathrm{UI} / \mathrm{ml}$ de plasma). En cas de SAPL obstétrical réfractaire malgré un traitement bien conduit associant aspirine et héparine à dose efficace, certains proposent l'adjonction de corticoïdes à faible dose (prednisolone $10 \mathrm{mg} / \mathrm{j}$ ), mais au prix d'une augmentation significative du risque de prématurité, d'hypertension artérielle et de diabète gestationnel, ou d'hydroxychloroquine (Plaquenil ${ }^{\circledR}$ ), y compris en cas de SAPL primaire [33,34]. Il a été démontré récemment que l'hydroxychloroquine jouait un rôle protecteur sur le bouclier que l'annexine $\mathrm{V}$ forme sur la couche de phospholipides cibles des APL. Cela lui confère potentiellement un rôle préventif dans la survenue de FCS [34]. Pour les patientes aux antécédents de SAPL vasculaire, avec une indication d'anticoagulation curative par anti-vitamine $\mathrm{K}$ (AVK) au long cours, on recommande de substituer ce traitement par héparine à dose curative dès le diagnostic de grossesse, du fait d'un risque tératogène entre 6 et $12 \mathrm{SA}$, et d'associer de l'aspirine 100 $\mathrm{mg} / \mathrm{j}$. Le port de bas de contention adaptés est essentiel dans ce contexte.

Surveillance de la grossesse : Une fois la grossesse diagnostiquée, nous recommandons un suivi multidisciplinaire associant un obstétricien et un spécialiste du SAPL. Le suivi est mensuel jusqu'à la moitié de la grossesse puis toutes les 2 semaines, et enfin, hebdomadaire jusqu'à l'accouchement. Les paramètres de surveillance sont résumés dans le tableau 3.

Tableau 3. Paramètres de surveillance de la grossesse au cours du SAPL.

Paramètres cliniques :

$\mathrm{PA}, \mathrm{BU}, \mathrm{OMI}$, poids, signes neurosensoriels, diurèse, signes de

poussée lupique (si SAPL secondaire à un LES), thrombose vasculaire. Paramètres biologiques:

NFS, plaquettes, LDH, haptoglobine, bilirubine indirecte, schizocytes, transaminases, créatininémie, uricémie, protéinurie de $24 \mathrm{~h}$, glycémie à jeun et HGPO si corticothérapie associée, sédiment urinaire et C3 si lupus associé.

\section{Paramètres échographiques:}

Échographie fœtale trimestrielle à 12,22 et 32 SA.

Doppler des artères utérins à 20 SA, et répété à 24 SA en cas

d'anomalie.

Si lupus associé avec anticorps anti-SSA ou anti-SSB:

échocardiographie fœtale tous les 15 jours entre 16 et 24 SA ou

hebdomadaire si antécédents de BAV ou de lupus néonatal.
Une surveillance régulière de la pression artérielle, du poids, des œdèmes, de la protéinurie, des plaquettes et des transaminases ou d'une éventuelle hémolyse permet de dépister précocement la prééclampsie et/ou le syndrome de HELLP. On recherche une poussée d'une éventuelle connectivite ou une thrombose maternelle. Outre les 3 échographies obstétricales recommandées, une surveillance échographique régulière est préconisée de manière hebdomadaire à partir de 32 SA jusqu'à l'accouchement, et plus tôt en cas d'insuffisance placentaire suspectée. La surveillance des dopplers des artères utérines et ombilicale est réalisée à $20 \mathrm{SA}$, et répétée à $24 \mathrm{SA}$ en cas d'anomalie. Un Doppler obstétrical anormal est un élément prédictif important d'accidents obstétricaux [27].

Accouchement et post partum : L'accouchement est habituellement programmé autour de 38 SA. Le mode d'accouchement est en fonction des conditions obstétricales. En cas de SAPL obstétrical pur, l'HBPM est arrêté avec la fenêtre la plus courte pour permettre l'analgésie péridurale et l'accouchement, puis reprise pour couvrir le risque thrombotique dans le post-partum, à dose préventive et pour une durée de 06 semaines [31]. Certains anesthésistes proposent un relais par HNF en fin de grossesse, qui, avec une demi-vie plus courte, permet de raccourcir la fenêtre d'arrêt de l'héparine [10]. Pour les patientes aux antécédents de SAPL vasculaire, avec une indication d'anticoagulation curative au long cours, on recommande la reprise d'héparine en post-partum immédiat avec relais par AVK le plus tôt possible avec le même objectif d'anticoagulation qu'avant la grossesse. L'allaitement sous AVK est possible, sous réserve d'un apport régulier de vitamine $\mathrm{K}$ chez l'enfant (2 $\mathrm{mg}$ par voie orale par semaine). La fluindione (Préviscan ${ }^{\circledR}$ ) est contre-indiquée du fait d'un passage dans le lait non négligeable et de modifications possibles des tests de coagulation chez les enfants allaités. Ces réserves ne s'appliquent pas à la warfarine (Coumadine ${ }^{\circledR}$ ) qui doit donc être privilégiée dans ce contexte [31].

\section{CONCLUSION}

Le pronostic materno-fœtal au cours du SAPL s'est considérablement amélioré ces dernières années. Cette amélioration s'explique par une meilleure connaissances de la pathologie, une meilleure anticipation (consultation préconceptionnelle) et par une prise en charge adaptée de ces grossesses considérées à haut risque. Différents protocoles thérapeutiques ont été proposés en fonction du type de SAPL (thrombotique, ou obstétrical pur) et des antécédents obstétricaux avec un avantage indéniable pour l'association de faibles doses d'aspirine et d'héparine.

Déclaration d'intérêts: les auteurs ne déclarent aucun conflit d'intérêt en rapport avec cet article. 


\section{RÉFÉRENCES}

1. Masliah-Planchon J, Darnige L. Antiphospholipid antibodies and haemostasis. Rev Med Interne 2012; 33:181-188.

2. Giannakopoulos B, Krilis SA. The pathogenesis of the antiphospholipid syndrome. N Engl J Med. 2013; 368:1033-1044.

3. Wilson WA, Gharavi AE, Koike T, et al. International consensus statement on preliminary classification criteria for definite antiphospholipid syndrome: report of an international workshop. Arthritis Rheum. 1999; 42:1309-1311.

4. Miyakis S, Lockshin MD, Atsumi $\mathrm{T}$, et al. International consensus statement on an update of the classification criteria for definite antiphospholipid syndrome. J Thromb Haemost. 2006; 4:295-306.

5. Solano C, Lamuño M, Vargas A, Amezcua-Guerra LM. Comparison of the 1999 Sapporo and 2006 revised criteria for the classification of the antiphospholipid syndrome. Clin Exp Rheumatol. 2009; 27:914-919.

6. Pourrat $\mathrm{O}$, Jollit $\mathrm{C}$, Gombert J-M, et al. Clinical relevance of the recent update of the classification criteria for definite antiphospholipid syndrome: an obstetric medicine clinic series of 107 patients. J Thromb Haemost. 2006; 4:2276-2277.

7. Costedoat-Chalumeau N, Guettrot-Imbert G, Leguern V, et al. Grossesse et syndrome des antiphospholipides. Rev Med Interne 2012; 33:209-216.

8. Rodriguez-Garcia JL, Bertolaccini ML, Cuadrado MJ, et al. Clinical manifestations of antiphospholipid syndrome with and without antiphospholipid antibodies (the so-called "seronegative APS"). Ann Rheum Dis. 2011; 71:242-244.

9. Bramham K, Hunt BJ, Germain S, et al. Pregnancy outcome in different clinical phenotypes of antiphospholipid syndrome. Lupus 2010; 19:58-64.

10. Le Guern V, Goffinet F. Grossesse et anticorps antiphospholipides. Presse Med. 2008; 37:1666-1675.

11. Nodler J, Moolamalla SR, Ledger EM, Nuwayhid BS, Mulla ZD. Elevated antiphospholipid antibody titers and adverse pregnancy outcomes: analysis of a population-based hospital dataset. BMC Pregnancy Childbirth 2009;9:11.

12. Huong DL, Wechsler B, Bletry $\mathrm{O}$, et al. A study of 75 pregnancies in patients with antiphospholipid syndrome. J Rheumatol. 2001; 28:20252030.

13. Yamada H, Atsumi T, Kobashi G, et al. Antiphospholipid antibodies increase the risk of pregnancy-induced hypertension and adverse pregnancy outcomes. J Reprod Immunol. 2009; 79:188-195.

14. Cervera R, Piette J-C, Font J, et al. Antiphospholipid syndrome: clinical and immunologic manifestations and patterns of disease expression in a cohort of 1,000 patients. Arthritis Rheum. 2002; 46:1019-1027.

15. Abou-Nassar K, Carrier M, Ramsay T, Rodger MA. The association between antiphospholipid antibodies and placenta mediated complications: a systematic review and meta-analysis. Thromb Res. 2011; 128:77-85

16. Branch DW, Porter TF, Rittenhouse L, et al. Antiphospholipid antibodies in women at risk for preeclampsia. Am J Obstet Gynecol. 2001; $184: 825-832$
17. Le Thi Huong D, Wechsler B, Piette JC. Pregnancy and systemic lupus erythematosus. Rev Med interne 2008; 29:725-730.

18. Le Thi Thuong D, Tieulie N, Costedoat $\mathrm{N}$, et al. The HELLP syndrome in the antiphospholipid syndrome: retrospective study of 16 cases in 15 women. Ann Rheum Dis. 2005; 64:273-278.

19. Appenzeller S, Souza FHC, Wagner Silva de Souza A, et al. HELLP syndrome and its relationship with antiphospholipid syndrome and antiphospholipid antibodies. Semin Arthritis Rheum. 2011; 41:517-523.

20. Cervera R, Font J, Gomez-Puerta JA, et al. Validation of the preliminary criteria for the classification of catastrophic antiphospholipid syndrome. Ann Rheum Dis. 2005; 64:1205-1209.

21. Gomez-Puerta JA, Cervera R, Espinosa G, et al. Catastrophic antiphospholipid syndrome during pregnancy and puerperium: maternal and fetal characteristics of 15 cases. Ann Rheum Dis.2007;66:740-6.

22. Cervera $R$, Bucciarelli $S$, Plasin MA, et al. Catastrophic antiphospholipid syndrome: descriptive analysis of a series of 280 patients from the "CAPS Registry". J Autoimmun 2009; 32:240-245.

23. Carp HJA, Shoenfeld Y. Antiphospholipid antibodies and infertility. Clin Rev Allergy Immunol. 2007; 32:159-161.

24. Kovács $\mathrm{M}$, Hartwig $\mathrm{M}$, Aleksza $\mathrm{M}$, et al. Antiphospholipid antibodies in relation to sterility/infertility. Hum Immunol. 2012; 73:726-731.

25. Ruffatti A, Tonello M, Visentin MS, et al. Risk factors for pregnancy failure in patients with antiphospholipid syndrome treated with conventional therapies: a multicentre, case-control study. Rheumatology 2011; 50:1684-1689.

26. Salmon JE, Kim M, Guerra M, et al. Absence of lupus anticoagulant is a strong predictor of uncomplicated pregnancy in patients with APL antibodies. Arthritis Rheum. 2008; 59:403.

27. Le Thi Huong D, Wechsler B, Vauthier-Brouzes D, et al. The second trimester Doppler ultrasound examination is the best predictor of late pregnancy outcome in systemic lupus erythematosus and/or the antiphospholipid syndrome. Rheumatology 2006; 45:332-338.

28. Rai $\mathrm{R}$, Cohen $\mathrm{H}$, Dave $\mathrm{M}$, Regan $\mathrm{L}$. Randomised controlled trial of aspirin and aspirin plus heparin in pregnant women with recurrent miscarriage associated with phospholipid antibodies. BMJ. 1997; 314:253-257.

29. Kutteh WH. Antiphospholipid antibody-associated recurrent pregnancy loss: treatment with heparin and low-dose aspirin is superior to low-dose aspirin alone. Am J Obstet Gynecol. 1996; 174:1584-1589.

30. Empson M, Lassere $M$, Craig J, Scott J. Prevention of recurrent miscarriage for women with antiphospholipid antibody or lupus anticoagulant. Cochrane Data base Syst Rev. 2005:CD002859.

31. Ruiz-Irastorza G, Crowther M, Branch W, Khamashta MA Antiphospholipid syndrome. Lancet 2010; 376:1498-1509.

32. Lebaudy C, Hulot JS, Amoura Z, et al. Changes in enoxaparin pharmacokinetics during pregnancy and implications for antithrombotic therapeutic strategy. Clin Pharmacol Ther. 2008; 84:370-377.

33. Bramham K, Thomas M, Nelson-Piercy C, et al. First-trimester low-dose prednisolone in refractory antiphospholipid antibody-related pregnancy loss. Blood 2011; 117:6948-6951.

34. Rand JH, Wu XX, Quinn AS, et al. Hydroxychloroquine protects the annexin A5 anticoagulant shield from disruption by antiphospholipid antibodies: evidence for a novel effect for an old antimalarial drug. Blood 2010; 115:2292-2299.

Cet article a été publié dans le « Batna Journal of Medical Sciences » BJMS, I'organe officiel de « I'association de la Recherche Pharmaceutique - Batna»

Le contenu de la Revue est ouvert « Open Access » et permet au lecteur de télécharger, d'utiliser le contenu dans un but personnel ou d'enseignement, sans demander l'autorisation de l'éditeur/auteur.

Avantages à publier dans BJMS :

Open access : une fois publié, votre article est disponible gratuitement au téléchargement

Soumission gratuite : pas de frais de soumission, contrairement à la plupart des revues « Open Access »

Possibilité de publier dans 3 langues : français, anglais, arabe

Qualité de la relecture : des relecteurs/reviewers indépendants géographiquement, respectant l'anonymat, pour garantir la neutralité et la qualité des manuscrits.

Pour plus d’informations, contacter BatnaJMS@gmail.com

ou connectez-vous sur le site de la revue : www.batnajms.com 